\title{
Versorgung mit Unterstützter Kommunikation - Perspektive der Sprachtherapie
}

\section{AAC Care from the Perspective of Speech and Language Therapy}

Barbara Giel, Andrea Liehs

\author{
Neue Versorgungsformen der Unterstützten Kommunikation (UK) als Bestandteil \\ der logopädischen Therapie werden im Rahmen eines Forschungsprojekts des \\ G-BA erprobt. Dabei wird klar, dass Veränderungen auf den Ebenen der Struktur-, \\ Prozess- und Ergebnisqualität notwendig sind, um eine flächendeckende und \\ qualitätsgesicherte UK-Versorgung zu ermöglichen. Im Bereich des Heilmittels \\ Sprachtherapie gilt es Qualitätskriterien zu definieren.
}

\section{LERNZIEL}

Der Lesende setzt sich mit der aktuellen Situation des Einsatzes von UK in der Sprachtherapie auseinander. Darüber hinaus lernt der Lesende Denkmodelle zur möglichen UK-Versorgung und Finanzierung im Gesundheitswesen kennen.

\section{Aktuelle Versorgungssituation in Deutschland}

Anders als im angloamerikanischen Sprachraum zählt der Bereich der Unterstützten Kommunikation (UK) nicht zu den curricular verankerten Ausbildungs- und Studieninhalten von Logopäden und Sprachtherapeuten. Historisch betrachtet ist die UK in Deutschland vor allem durch die Sonderpädagogik etabliert worden. Die Förderung der Kommunikation wurde in der „Leistungsbeschreibung Sprachtherapie“ der Heilmittelrichtlinien in verschiedenen Indiktionsschlüsseln (u.a. SP1, SP5) ausdrücklich aufgeführt.

In nationalen Forschungsstudien wurde aufgezeigt, dass UK in unterschiedlichen Facetten in der Logopädie in Deutschland angekommen ist [1,2]. Aufgrund unterschiedlicher beruflicher Sozialisationen in der Sonderpädagogik und in der Logopädie existieren verschiedene Zugänge zu dem Themenbereich UK, was sich u. a. in zugrundeliegenden Theorien/Modellen und im Gebrauch von Terminologie wiederfindet [3]. Betrachtet man die letzten 20 Jahre in Deutschland, so ist ein deutlicher Anstieg an Veröffentlichungen zu dem Themenbereich UK in der Logopädie zu beobachten. Dort wird aufgezeigt, wie bei den verschiedensten
Indikationen in der Logopädie mit UK-Methoden gearbeitet werden kann [4-6].

Aufgrund der Komplexität von UK stellt sich die Frage, wie eine qualitätsgesicherte UK-Versorgung in Deutschland aussehen könnte und welche Rolle dabei die Logopädie spielt. Die Unterschiede und Gemeinsamkeiten zwischen einer komplexen UK-Versorgung, UK-Förderung, UK-Therapie und/oder Sprachtherapie mit UK-Methoden sind aktuell.

Die UK-Versorgung ist in Deutschland aktuell nicht einheitlich geregelt, und je nach Bundesland und Institution sind unterschiedliche Berufsgruppen und verschiedene Wege der Finanzierung anzutreffen. Menschen mit UK-Bedarf finden oftmals nur auf Umwegen eine firmenunabhängige und qualitätsgesicherte UK-Beratung. Darüber hinaus ist in Deutschland eine weiterführende (sprach-)therapeutische Versorgung flächendeckend noch nicht gewährleistet. Angehörige, Betroffene und Helfersysteme berichten häufig von einer aufwändigen und zeitintensiven Suche nach den geeigneten UK-Spezialisten und UK-Angeboten. Um diese Versorgungslücke zu schließen und klare Versorgungswege aufzuzeigen, finden in Deutschland aktuell entsprechende Forschungsprojekte statt.

\footnotetext{
Merke

Es fehlt eine flächendeckende und qualitätsgesicherte UK-Versorgung in Deutschland.
}

Der Gemeinsame Bundesausschuss (G-BA) hat den Auftrag neue Versorgungsformen, die über die bisherige Regelversorgung der gesetzlichen Krankenversicherung hinausgehen und auf einen Erkenntnisgewinn zur Verbesserung der bestehenden Versorgung ausgerichtet sind, zu fördern. Unter der Leitung der Forschungs- 
und Beratungsstelle für UK (FBZ-UK) der Universität zu Köln wurde ein Forschungsprojekt initiiert, in dem der Frage nachgegangen wird, wie eine qualitätsgesicherte Versorgung und deren Versorgungspfade in der UK in Deutschland aussehen könnten.

Am sogenannten MUK-Projekt (Maßnahmen der Unterstütze Kommunikation) nehmen verschiedene Konsortialpartner (UK-Beratungsstelle Köln, Zentrum für UK Moers, Mittendrin Hamburg) teil, die während der Projektlaufzeit neue UK-Versorgungsformen erproben. Das „Ziel des Projekts ist es (...), die personalisierte Versorgung zu verbessern und die Lücken in der Versorgungskette zur ,Unterstützten Kommunikation‘ zu schließen. Hierzu wird ein bereits bestehender Selektivvertrag um ein personalisiertes und organisiertes Fallmanagement, Patiententraining sowie einen individualisierten Therapieplan erweitert und umgesetzt.“ [12].

Durch das MUK-Projekt sollen qualitätsgesicherte Versorgungswege und -strukturen in Deutschland etabliert werden [7]. Ein Entwurf zur Qualitätssicherung auf den Ebenen der Struktur-, Prozess- und Ergebnisqualität wurde bereits vorgelegt. Dieser geht von einer hierarchischen Struktur aus, in der zertifizierte UK-Beratungsstellen und UK-Kompetenzstellen von einer nationalen Zentralstelle koordiniert werden [7]. Das Ziel ist ein flächendeckendes und qualitätsgesichertes UKVersorgungsangebot in Deutschland.

Im MUK Projekt werden Patientinnen und Patienten unter anderem mit einer sogenannten UK-Therapie versorgt. Es gilt zu klären, welche Heilmittelerbringer diese Therapie in Zukunft erbringen könnten, und welche Voraussetzungen zum Erbringen dieser Leistung notwendig sein werden. Zum aktuellen Zeitpunkt existieren im System der GKV (Heilmittelrichtlinien) keine gesonderten Abrechnungspositionen für die aufwändige UK-Therapie und/oder Sprachtherapie mit UK-Methoden.

Qualitätskriterien, die die Güte dieser Leistungen sicherstellen, sollen im Rahmen des MUK-Projekts entwickelt werden. An verschiedenen Stellen wurde bereits darauf hingewiesen, dass ein umfassendes Wissen sowie eine umfangreiche Methodenkompetenz nötig sind, um eine entsprechende Qualität in der UK-Therapie gewährleisten zu können [6-8].

\section{Rolle der Sprachtherapie/Logopädie in der interdisziplinären Versorgung von Schulkindern}

Die fehlenden einheitlichen Qualitätsstandards im Bereich der UK sind eng verbunden mit der Frage, was in Deutschland die Aufgabe und die Rolle der einzelnen Fachdisziplinen und die der Eltern/Angehörigen in der UK-Versorgung sein könnten. Im deutschen Bildungsund Gesundheitssystem sind aktuell keine (einheitlichen) Regelungen in Bezug auf die Zuständigkeiten und die Vergütung von Leistungen innerhalb der Versorgung von Kindern mit UK-Bedarf zu finden.

Der oben erwähnte Innovationsfonds und das vom GBA bewilligte MUK-Projekt beziehen sich im Schwerpunkt auf die UK-Leistungen, die im Gesundheitssystem verortet sind oder sein sollten. Es steht außer Frage, dass viele Fachdisziplinen aus den Bereichen Bildung und Pädagogik an gelingender Kommunikation beteiligt sind. Aktuell ist die Frage der Zuständigkeiten für die UK-Versorgung mit therapeutischen und pädagogischen Maßnahmen nicht eindeutig geklärt.

Gibt es beispielsweise in Nordrhein-Westfalen an jeder Förderschule für körperlich motorische Entwicklung (KME) und geistige Entwicklung (GG) sogenannte UKBeauftragte, die in der Regel mit einem sehr geringen Stundendeputat ausgestattet sind, so ist die inhaltliche Gestaltung dieser Aufgabe nicht oder kaum definiert. Darüber hinaus sind große Unterschiede in den einzelnen Förderschulformen in Bezug auf das Thema UK zu finden. An einigen Förderschulen für körperlich motorische Entwicklung (KME) sind beispielsweise UK-Beratungsstellen angegliedert, wobei es in Deutschland zurzeit noch keine Qualitätskriterien für solche Stellen gibt, sodass die materielle, personelle, zeitliche und inhaltliche Ausstattung dieser Beratungsstellen nicht einheitlich geregelt ist.

Dies führt im Versorgungsablauf von Menschen mit UKBedarf zu Verunsicherung. Eltern, Ärzte und weitere Fachpersonen sind häufig nicht darüber informiert, wo und wie firmenunabhängige UK-Beratungen stattfinden können. Nach einer Beratung ist dann qua Gesundheits- und Bildungssystem nicht eindeutig geklärt, wer mit welchem Auftrag welche Aspekte der UK-Versorgung übernimmt, im Alltag implementiert und wer für die Nachhaltigkeit/Evaluation der Versorgung zuständig ist ( $>$ Abb. 1). 


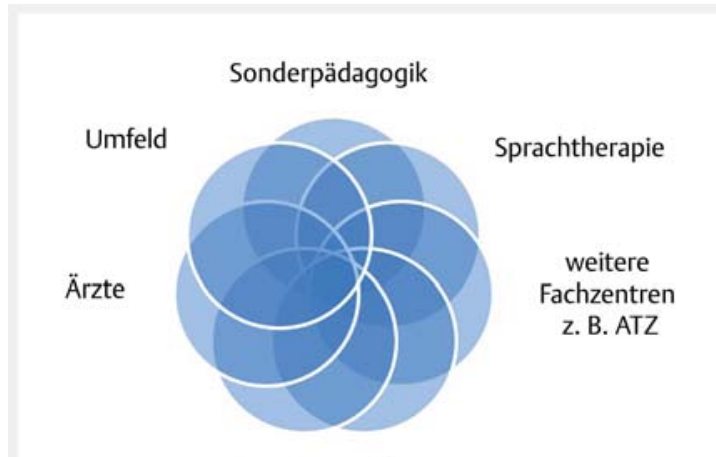

ggfs. Hilfsmittelfirmen

- Abb. 1 Verschiedene Akteure in der UK-Versorgung am Beispiel Schulkind mit meist ungeklärter Zuständigkeit.

\section{Unterstützte Kommunikation in der Sprachtherapie}

In verschiedenen Veröffentlichungen und Konzepten werden UK-Maßnahmen als Bestandteil der Sprachtherapie angesehen [4-6]. Kaiser-Mantel beschreibt das Dilemma, dass UK und Sprachtherapie sowohl bei Therapeuten als auch bei Betroffenen, Bezugspersonen und Institutionen oftmals als „Entweder-Oder-Leistung “ verstanden wird. Dabei kommt UK vielfach erst zum Einsatz, wenn andere Methoden der Logopädie keinen Erfolg gezeigt haben. Kaiser-Mantel fordert, die UK „sollte als Methode innerhalb der Sprachtherapie betrachtet werden, um dem Kind mit (noch) eingeschränkter oder (noch) nicht vorhandener Lautsprache Kommunikation und Sprache zu ermöglichen “ [5].

Insbesondere vor dem Hintergrund der ICF ist der Einsatz alternativer und ergänzender Kommunikationsformen unabdingbar, wenn die vorhandenen lautsprachlichen Fähigkeiten noch nicht oder nicht mehr ausreichen, um eine Partizipation zu gewährleisten. Bei bestimmten Störungsbildern ist der Fokus von einer rein funktionsorientierten Therapie zu lösen. Es gilt durch den Einsatz von UK die kommunikative Teilhabe zu sichern.

Nonn weist darauf hin, dass insbesondere ein frühzeitiger Einsatz von UK bei Kindern mit komplexen Behinderungen von Relevanz ist, da eine eindeutige Entwicklungsprognose in den ersten Lebensjahren vielfach nicht gestellt werden kann. Hier sollte die UK ein wesentlicher Bestandteil der Logopädie sein, um eine alleinige Ausrichtung auf Lautsprache zu vermeiden und ergänzende Kommunikationsformen zur Verfügung zu stellen, um viele Kommunikationswege zu öffnen [4].
Im Störungsbereich der erworbenen Sprach- und Sprechstörungen ist nach Liehs und Giel eine „sprachtherapeutische Diagnostik und Therapie geboten, die die ICF-Komponenten der Funktion, Aktivität und Partizipation beinhaltet. Dies impliziert eine funktionelle Betrachtung auf der einen Seite und eine kommunikativ-pragmatische Betrachtung auf der anderen Seite“ [9]. So kann die Funktionstherapie im Rahmen der logopädischen Versorgung von Menschen mit fortschreitenden Erkrankungen die Lautsprache zunächst stützen, den Verlust der Lautsprache wird sie allerdings nicht verhindern. Nur durch den Einsatz von Maßnahmen der UK wird eine kommunikative Teilhabe gesichert werden. Umgekehrt kann im Rahmen eines akuten Sprach- und/Sprechverlusts UK solange Kommunikation ermöglichen, bis eine ausreichende Lautsprachfunktion wiederhergestellt werden konnte. In den letzten Jahren hat sich gezeigt, dass eine gelingende Kommunikation mit Mitteln der UK nur möglich ist, wenn das Umfeld des UK-Nutzenden in entsprechende soziale Interaktion geht.

Sprachtherapie muss an dieser Stelle erfassen, was auf der Ebene der Funktion sowie der individuellen kommunikativen Fähigkeiten zu erreichen ist und wo der Einbezug des sozialen Umfeldes notwendig wird. Zum Übertrag von UK in den Alltag und zur Aktivierung der jeweiligen Ressourcen wurden die Moderierten Runden Tische (MoRTi) als positives Vorgehen evaluiert [10, 11]. Durch ein Zusammentreffen aller am Kommunikationsprozess beteiligten Personen kann eine Veränderung der Sichtweise auf Kommunikation und den UKEinsatz gefördert werden.

Auf die Bedeutung der Bezugspersonen und die Zusammenarbeit vieler verschiedener Fachdisziplinen in der UK-Versorgung weisen auch Lüke und Vock [6] hin. Sie entwickelten eine „spezifische Strukturierungshilfe für SprachtherapeutInnen“ zur interdisziplinären UKVersorgung. Unter KEMKUS fassen sie den „Konzeptuellen Einbezug von Methoden der Unterstützen Kommunikation in der Sprachtherapie“ zusammen und zeigen ein Versorgungsmodell aus Diagnostik, der Erprobung und Festlegung von Interventionsmöglichkeiten unter Einbezug des sozialen Umfelds, der Zielformulierung und Etablierung der UK-Maßnahme auf. Lüke und Vock heben die Rolle der Bezugspersonen besonders hervor und fordern, dass diese bereits „von Beginn der Therapie an aktiv in den Interventionsprozess einbezogen werden. Kommunikation findet immer zwischen Menschen statt. Der Erfolg jeder Intervention zur Verbesserung der Kommunikationssituation von Menschen mit eingeschränkten Mitteilungsmöglichkeiten ist daher nicht zuletzt auch den Angehörigen dieser Personen abhängig“ [6]. Für die sprachtherapeutische Versorgung bedeutet dies Angehörige in die Therapie 
einzubeziehen und ihnen Kommunikations- und Modellingstrategien für den Alltag zu vermitteln.

\section{Merke}

UK ist Bestandteil der logopädischen Versorgung. Es wird ersichtlich, wie komplex die Sprachtherapie unter Einbezug der UK ist, und dass das aktuelle Heilmittel Sprachtherapie die dafür notwendigen Leistungs- und Abrechnungspositionen nicht zur Verfügung stellt.

\section{Bedingungen für ein qualitäts- gesichertes Heilmittel im Rahmen der UK}

Um in Deutschland eine flächendeckende und qualitätsgesicherte UK-Versorgung zu gewährleisten, sind Veränderungen auf Ebene der Struktur- und Prozessqualität notwendig. Zunächst gilt es, ein explizites UKFachwissen qualitätsgesichert zu erwerben. Dazu gibt es zurzeit noch keine Aus- oder Weiterbildungsstandards. Aufgrund der noch fehlenden Verankerung von UK in den Ausbildungsgängen der Sprachtherapie/Logopädie sind spezifische Weiterbildungen (u.a. Lehrgang Unterstützte Kommunikation (LUK), UK-Coach ${ }^{\odot}$, Fachkraft Unterstützte Kommunikation nach Standard der GfUK e. V.) entwickelt worden. Diese interdisziplinär angelegten Weiterbildungen haben zum Ziel, möglichst vielen Fachdisziplinen UK-Wissen zu vermitteln.

Da jedoch Sprachtherapeuten aufgrund ihrer Ausbildung bereits über ein breites Wissen im Bereich Sprache und Kommunikation verfügen, bringen sie sowohl andere Voraussetzungen als auch andere inhaltliche Bedarfe an eine solche UK-Weiterbildung mit [8]. Neben der Vermittlung von UK-Wissen müssen die Diagnostik mit Hilfe von Unterstützten Kommunikationsformen, die Interventionsplanung auf der Basis der Indikationsschlüssel des Heilmittelkataloges und die Integration von UK-Formen in bestehende logopädische Konzepte zentrale Bestandteile einer UK-Weiterbildung sein.

Um diese Lücke in Deutschland zu füllen, wurde die Weiterbildung „FachtherapeutIn Unterstützte Kommunikation“ speziell für Fachpersonen aus Logopädie und Sprachtherapie entwickelt. Diese Weiterbildung wird als Beitrag zur Qualitätssicherung in der UK-Versorgung in Deutschland verstanden. Aufbauend auf den Inhalten der logopädischen Curricula wird in 8 Modulen ein umfangreiches UK-spezifisches Wissen theoretisch und praktisch vermittelt. Bestandteile der Weiterbildung sind außerdem ein fortlaufender Kurs in lautsprachunterstützenden Gebärden (LUG) sowie die Vermittlung verschiedener Beratungskompetenzen [8].
Obwohl in der Gesamtbetrachtung der sprachtherapeutischen Versorgung in Deutschland die hochkomplexen UK-Versorgungen einen eher geringen Prozentsatz ausmachen, ist hier nach geeigneten Versorgungswegen zu suchen. Durchgeführte Befragungen sowie die alltägliche Praxis zeigen, dass das bestehende Heilmittel Sprachtherapie aktuell nicht ausreichend materielle, zeitliche und finanzielle Rahmenbedingungen und Ressourcen zur Verfügung stellt, um eine umfassende Therapie mit Methoden der UK durchzuführen $[1,2]$. Die fehlende Vergütung der zeit- und materialaufwändigen Diagnostik und Therapie, die Notwendigkeit der Bereitstellung kostenintensiver UK-Materialien, fehlende Leistungspositionen für die interdisziplinäre Zusammenarbeit und Gutachtenerstellung können hier beispielhaft genannt werden.

In welcher Form die Leistung UK-Therapie in den Heilmittelrichtlinien verankert werden könnte, muss diskutiert werden. Das MUK-Projekt des Innovationsausschusses kann dazu einen wichtigen Beitrag leisten. Da die Logopädie die einzige Expertin für Sprach-, Sprech-, Stimm- und Schluckstörungen ist, bedarf es innerhalb dieses Fachgebiets der Beschreibung der Leistung „Unterstützte Kommunikation“. Im Rahmen der Leistungsbeschreibung können Bedingungen definiert werden, die der Sprachtherapie ermöglicht, eine qualitätsgesicherte Leistung zu erbringen sowie Patienten einen leichten und evidenzbasierten Zugang zur UK zu ermöglichen.

\section{FAZIT}

Die UK findet in der logopädischen Therapie durchaus Beachtung; innerhalb verschiedener Konzepte wird die UK immer wieder als Bestandteil der Sprachtherapie gesehen. Es wird aber auch deutlich, dass die UK-Versorgung keineswegs flächendeckend und qualitätsgesichert erfolgt. Im Rahmen eines aktuell laufenden Forschungsprojekts des G-BA werden neue Versorgungsformen im Kontext UK erprobt. Es ist ersichtlich, dass es Veränderungen auf den Ebenen der Struktur-, Prozess- und Ergebnisqualität bedarf und diese im Bereich des Heilmittels Sprachtherapie verankert werden müssen.

\section{Schlüsselwörter}

UK-Versorgung, UK-Therapie, interdisziplinäre Versorgung, Logopädie 


\section{Interessenkonflikt}

Die Autorinnen/Autoren geben an, dass kein Interessenkonflikt besteht.

\section{Autorinnen/Autoren}

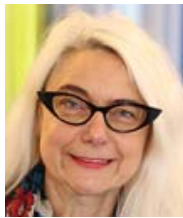

\section{Barbara Giel}

Dr. Barbara Giel ist akademische Sprachtherapeutin, Familientherapeutin und systemische Supervisorin/Organisationsberaterin. Sie leitet das Zentrum für Unterstützte Kommunikation (ZUK) Moers, ist fachliche Leitung des Zentrums für Sprachtherapie Moers und Mitglied des Beratungsnetzwerk soulutions. Seit 1992 Lehraufträge, Fortbildungen sowie Veröffentlichungen u.a. zum Thema UK, systemische Beratung und Qualitätsmanagement.

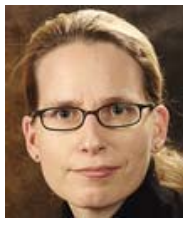

\section{Andrea Liehs}

Dr. Andrea Liehs ist akademische Sprachtherapeutin und als fachliche Leitung im Zentrum für Sprachtherapie Moers tätig. Seit 2000 Fortbildungen, Lehraufträge und Veröffentlichungen zum Thema UK.

\section{Korrespondenzadresse}

Dr. Barbara Giel

Zentrum für Unterstützte Kommunikation (ZUK Moers)

Goethestr. 16

47441 Moers

E-Mail: giel@sprachtherapie-moers.de

\section{Literatur}

[1] Förster Chr, Wahl M. Unterstützte Kommunikation in der Sprachtherapie: Interdisziplinär bewirken wir mehr. Spektrum Patholinguistik 2019; 11: 91-111. Im Internet: https://doi.org/10.25932/publishup-43776
[2] ThorndahI D. Online-Befragung zu UK in der Logopädie. Studie zu Barrieren und Gelingensbedingungen bei der Umsetzung von Unterstützter Kommunikation. Forum Logopädie 2020; 34: 32-33

[3] Giel B. Unterstützte Kommunikation und Sprachtherapie/ Logopädie - zwei komplementäre Systeme! Logos 2014; 22: 201-207

[4] Nonn K. Unterstützte Kommunikation in der Logopädie. Stuttgart: Thieme; 2011

[5] Kaiser-Mantel H. Unterstützte Kommunikation in der Sprachtherapie. München: Reinhardt; 2012

[6] Lüke C, Vock S. Unterstützte Kommunikation bei Kindern und Erwachsenen. Berlin: Springer; 2019

[7] Bernasconi T, Boenisch J, Giel B, Sachse ST. Qualitätssicherung und Standards in der UK-Versorgung. In: Boenisch J, Sachse ST. Kompendium Unterstützte Kommunikation. Stuttgart: Kohlhammer; 2020: 386-396

[8] Giel B, Hellrung U. Logopädie und Unterstützte Kommunikation. Die Rolle der Logopädie in der Versorgung von Menschen ohne Lautsprache. Forum Logopädie 2019; 5 : 20-24

[9] Liehs A, Giel B. UK-Therapie bei Erwachsenen mit erworbenen Kommunikationsstörungen. In: Boenisch J, Sachse ST. Kompendium Unterstützte Kommunikation. Stuttgart: Kohlhammer; 2020: 280-286

[10] Giel B. MoRTi: Moderierte Runde Tische bei komplexen Sprach- und Kommunikationsstörungen. In: Sprachförderung und Sprachtherapie. Dortmund: Verlag modernes lernen; 2017: 231-236

[11] Giel B, Liehs A, Preißler P et al. Qualitätssicherung in der UK Beratung durch Moderierte Runde Tische (MoRTi). uk \& forschung 2018; 2: 4-12

[12] Im Internet (zuletzt abgerufen am 20.04.2020): https:// innovationsfonds.g-ba.de/projekte/neue-versorgungsformen/muk-erweiterung-des-selektivvertrags-zu-massnahmen-der-unterstuetzten-kommunikation.102

Bibliografie

DOI https://doi.org/10.1055/a-1161-1240

Online-Publikation: 31.7.2020 | Sprache · Stimme · Gehör 2020; 44: 145-149

(c) Georg Thieme Verlag KG Stuttgart · New York ISSN 0342-0477 